\title{
SCMC: An Efficient Scheme for Minimizing Energy in WSNs Using a Set Cover Approach
}

\author{
Ahmed Redha Mahlous \\ Computer Science Department, Prince Sultan University, Riyadh 11586, Saudi Arabia, armahlous@psu.edu.sa; \\ Tel.: +966-50-845-0765
}

Received: 1 November 2017; Accepted: 8 December 2017; Published: 13 December 2017

\begin{abstract}
Energy-efficient clustering and routing are well known optimization problems in the study of Wireless Sensor Network (WSN) lifetime extension. In this paper, we propose an intelligent hybrid optimization algorithm based on a Set Cover approach to create clusters, and min-cost max-flow for routing (SCMC) to increase the lifetime of WSNs. In our method we used linear programming (LP) to model the WSN optimization problem. This model considers minimizing the energy for all nodes in each set cover (cluster), and then minimizing the routing energy between the nodes and the base station through intermediate nodes, namely cluster heads. To evaluate the performance of our scheme, extensive simulations were conducted with different scenarios. The results show that the set cover approach combined with the min-cost max-flow algorithm reduces energy consumption and increases the network's lifetime and throughput.
\end{abstract}

Keywords: set cover; min cost flow; cluster; optimization; wireless sensor network; routing

\section{Introduction}

The term "Wireless Sensor Network" (WSN) refers to a large number of spatially distributed autonomous nodes that organize themselves into a multi-hop wireless network for the monitoring and recording of physical or environmental conditions. Their applications include battlefield surveillance, target tracking, security, environmental control, habitat monitoring, source localization, fire detection, oil and gas pumping, and many more.

A WSN system consists of distributed nodes and a gateway that provides wireless connectivity back to the wired world (see Figure 1). They can be organized into three types of network topology as shown in Figure 2.

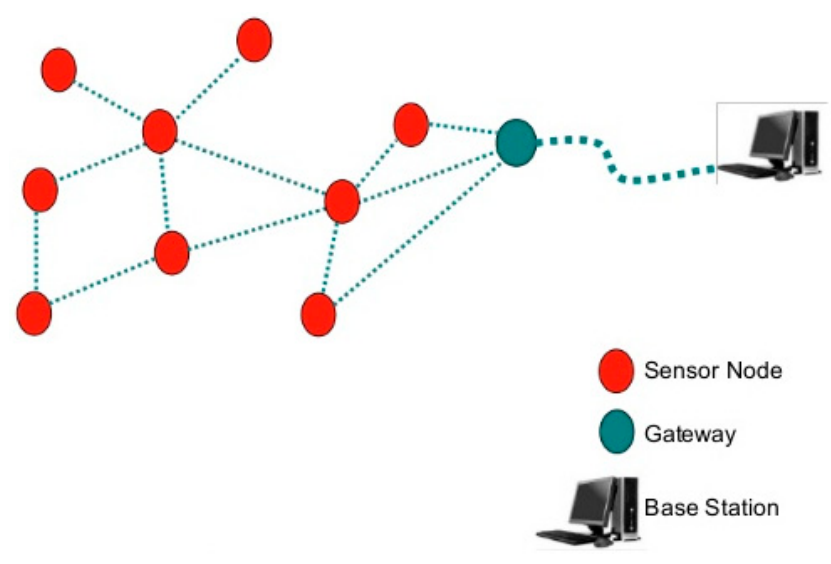

Figure 1. Wireless Sensor Network (WSN) Components, Gateway, and Distributed Nodes. 


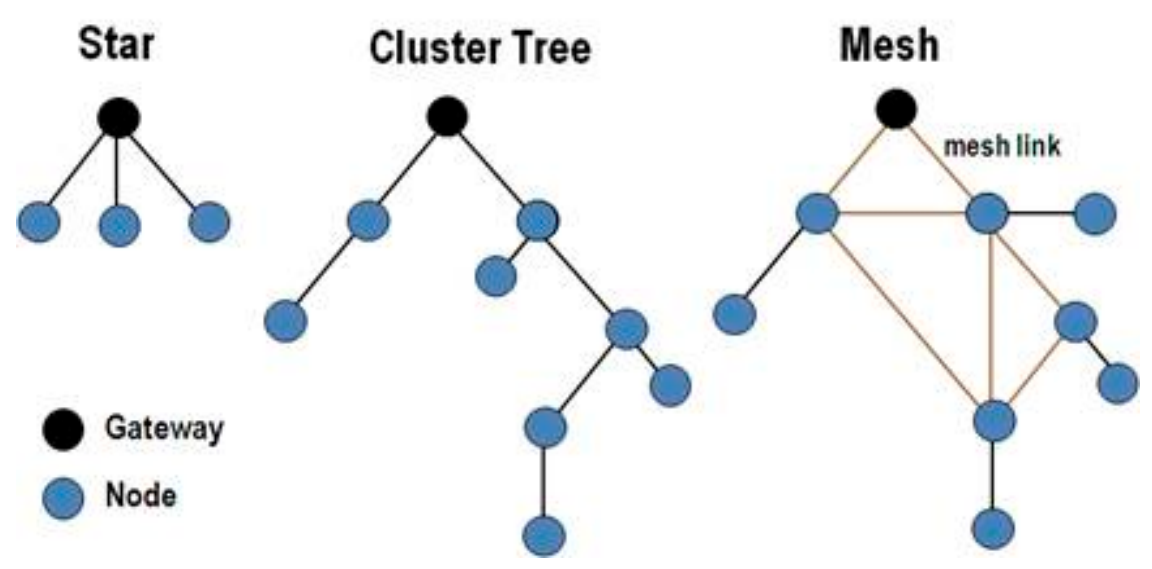

Figure 2. WSN Topologies.

A star network topology consists of a single base station and multiple remote nodes. The nodes cannot communicate directly with each other, while the base station can communicate with all of the nodes. In a mesh network topology, a node can directly communicate with any other node within its transmission range. This solves the single point of failure problem of star networks by allowing what is called multi-hop communication. In a cluster network topology, nodes are grouped to form a cluster with one of the nodes elected as a "cluster head" $(\mathrm{CH})$. Cluster topologies when employed in WSNs generally result in lower energy consumption and an increased overall lifetime of the network.

Because they are usually deployed in harsh environmental scenarios, WSN nodes rely solely on their batteries most of the time. As batteries have a finite capacity, minimizing energy consumption in order to extend the lifetime of sensor nodes without compromising their functionality is an important area of study in WSN research [1-5].

In WSNs, all nodes usually share common sensing tasks, each covering a different target area. This implies that not all sensors that are deployed within the same target area are required to perform their task continuously throughout the system's entire lifetime. Sensor nodes consume more energy when they are in transmission mode (active) than when they are in sleeping mode (inactive). If all sensor nodes within a coverage target area are active, the data collected will be redundant and a large amount of energy will be consumed and wasted. To save the overall energy of WSNs, our approach was to activate some sensor nodes while turning off others within each target area (set cover/cluster) without losing or disrupting system connectivity.

Specifically, the proposed model aims to determine a minimum set cover within a WSN, and to elect a relay node (cluster head) that will forward sensor node messages to the base station. Moreover, the model addresses the problem of finding the minimum cost routes from sensor nodes to the base station through the elected cluster heads using a min-cost flow algorithm. To achieve the best solution for expanding a WSN's lifetime, we propose a modified version of the min-cost flow algorithm by taking residual energy as a constraint value to judge different paths and choose the best one among them, i.e., the one with the highest residual energy.

While most of the approaches found in literature employ a Particle Swarm Optimization (PSO) algorithm to find clusters [6-10], in this paper we present a novel approach based on a set cover algorithm for finding clusters and a min-cost max-flow algorithm to find paths with minimum cost (energy consumption) and maximum residual energy. There are two main motivations for finding minimum set covers and using min-cost max-flow routing. The first is the need for an efficient energy scheme in WSNs, where sensor nodes tend to use small batteries for energy supply, and which are in many cases non-replenishable. Therefore an efficient activation management scheme is needed to conserve their energy, and thus that of the whole network. The second is the need for reliability in wireless networks. This need stems from the unpredictable nature of the wireless environment, which unlike its wired counterpart is more prone to link failures due to dead sensor nodes. A typical scenario 
where our approach can be of particular interest is if we consider a monitoring application targeted at the structural integrity of buildings, with WSN nodes deployed for data collection purposes over key city constructions such as roads, buildings, and bridges. When one critical event is detected (e.g., a dangerous flexure of a column), the monitoring application should trigger an alert to be delivered immediately to the WSN's data collection points (base station). In this case, it is particularly important to increase resiliency against link failures and ensure a long lifetime, which is what the SCMC technique presented in this paper achieves.

The remainder of the paper is organized as follows: Section 2 presents related work, Section 3 presents the approach, Section 4 describes simulation results, and Section 5 concludes the paper.

\section{Related Works}

Three key considerations in WSNs are coverage [11], connectivity [12], and battery lifetime [13]. Coverage refers to how well an area is monitored, while connectivity means that each node or device should be connected such that the data is sent back to the base station (BS). Any applied heuristic needs to fulfill the requirement of $Q$-coverage and P-connectivity, where $Q$ is the minimum number of sensors monitoring any one point in the area, and $\mathrm{P}$ is the number of disjoint paths between any two sensors.

In this section, we shall take a brief tour of the past approaches at solving these issues, starting with [14] in which the authors proposed a heuristic method called QC-MCSC to maximize the network lifetime while satisfying both the coverage and connectivity requirements. This method consists of three phases or layers where the output of one phase is the input of the next one. The first phase is the Coverage phase, which outputs the set of sensors that cover all the target areas. Next is the Connectivity and Redundancy Reduction phase, which takes the set of the first phase and applies the Breadth-First Search (BFS) algorithm to find the shortest path for each sensor. In this phase, unnecessary nodes are removed and any extra nodes are only added if the network is not fully connected, and the output is a minimal connected set cover of nodes. Finally, the Energy and Priority Updating phase determines the lifetime constant of each set cover from the second phase. The lifetime constant is the minimum lifetime available from sensors in a set cover. In this phase, if the battery life ' $\mathrm{B}$ ' of one node is less than the energy needed for communicating and sensing ' $E$ ', the node is removed from the set cover. Through simulation, the authors compared the Q-Coverage Maximum Connected Set Cover (QC-MCSC) algorithm with the Three Phase Iteration Connected Set Cover (TPICSC) algorithm [15] and the results showed that the former achieves a higher lifetime. Furthermore, the results of the simulations reveal that the proposed heuristic (QC-MCSC) is very close to the actual optimal result.

A difficulty that researchers face when they want to expand the coverage time span is the question of how to distribute the sensors into separate independent subdivisions, known as Disjoint Set Covers (DSC). This problem is identified as having an NP-hard complexity, and many researches have used the Boolean sensing model to solve it.

To facilitate topic lookup for the reader, we shall broadly divide the remaining studies by the type of approach and/or the specific WSN issue being addressed.

\subsection{Target Coverage}

A number of studies have focused on addressing the target coverage problem. The objective of the authors in [16] for example, was to have the maximum number of targets monitored by sensor nodes before they run out of energy. To extend the network lifetime, they proposed an approach to calculate the maximum number of disjoint sensor covers with a heuristic greedy algorithm using three parameters: (1) the set covers; (2) the number of targets; (3) the number of sensors. The proposed approach finds the set covers that can be used to maximize the network lifetime of a WSN in polynomial time. They showed that it gives a local maximum solution due to its heuristic search.

Maintaining full connectivity of the WSN was the primary focus of [17], in which the authors proposed an algorithm for ensuring that both conditions (i.e., coverage and connectivity) are met for 
conserving energy and extending the lifetime of the WSN. It relies on a distributed response procedure to locate the routes between the sensors and the sinks. The procedure allows for several routes leading from a sink to individual sensors to be discovered. Depending on the set of routes, a Minimum Distance Path (MDP) greedy algorithm is derived from resolving the Maximum Set Cover (MSC) issue with the goal of locating the highest possible number of set covers. The MDP-MSC algorithm works with the concept of choosing the sensors possessing the shortest distance to the set cover under construction, in order to include them into the contemporary set cover after each process. In this algorithm, the discovered routes, which are determined by the maximum number of routes per cover, influence the choice of sensors that are added to the set cover. The authors stated that the optimum value of the maximum number of routes per cover, as well as the level of density of the sensors within the target area, all impact the performance and control of transmission overhead in the WSN.

Based on simulation and experimental analysis using MDP-MSC, a number of set covers received a noticeable growth by $13 \%$ and $21 \%$ compared to the Greedy-MSC [18] and Heuristic Approach-Multidimensional Scaling (HA-MDS) algorithms [19]. MDP-MSC did not just outperform Greedy-MSC and HA-MDS; it also had the lowest time complexity between them. MDP-MSC achieved a balanced consumption of energy and less computational time.

On the other hand, the authors in [20] proposed a distributed lifetime coverage optimization (DiLCO) protocol to preserve coverage as well as boost the WSN's lifetime. The protocol begins by applying a divide and conquer algorithm to split the target location into smaller regions known as sub-locations, after which it is concurrently carried out in every single sub-location. Assumptions are made that the partitioning of the sub-locations is carried out in a regular manner and that the sensors are set uniformly in each sub-location. The periodic DiLCO protocol possesses four stages in each period: information exchange, leader election, decision, and sensing. Every period possesses a single set cover handling the sensing operation. This type of cyclic scheduling boosts the reliability of the WSN against sensor failures. Sensors without sufficient power to successfully carry out a period or those that fail before the decision phase is completed are not included in the scheduling procedure. If a sensor fails after being chosen, the quality is only reduced for the duration of the period as another functioning sensor is selected in the next period.

Two types of packets were used when carrying out the DiLCO protocol: info and Active/Sleep. Info packets are transmitted from a sensor to all others within the same sub-location with the purpose of providing information. Active/Sleep packets are transmitted by the leader to the other sensors within the same sub-location to notify them to set their status to either Active or Sleep in the sensing stage.

The challenges faced in implementing the DiLCO protocol lie in selecting the most appropriate leader in every single sub-location and the most suitable collection of active sensors to ascertain a high standard in coverage.

In [21], the aim was to find the maximum number of sensor coverage sets. The authors used two centralized algorithms and one localized algorithm. The two centralized algorithms deal with how the sensors are added to make a connected cover. The localized algorithm is used for communication between the local nodes. All three algorithms were only run once during the initialization phase. They concluded that keeping one coverage set active in a round-robin fashion would extensively reduce the energy consumption.

\subsection{Node Activation}

With regards to node activation, many existing studies propose alternating nodes between Sleep and Active modes, or regulating the sensing range of the nodes. The authors in [22] however, proposed to use both approaches together by setting up the minimum sensing radius for each sensor in the Active state. This reduces the active node's density, thereby reducing useless Media Access Control (MAC) layer interference, which would otherwise consume lots of power. 


\subsection{Node Distribution}

The authors in [23] proposed to choose the smallest possible number of nodes that will cover the critical targets by using a cost function called the Critical Control Factor. This function is mapped into 3 loops: (1) sensor availability check (adding the cover sets); (2) uncovered target check (populating the added cover sets with sensors); and (3) sensor applicability check (sorting the nodes and choosing the top-scoring node). The main benefits of this proposed solution are that it is flexible, has a low complexity, and has a short execution time. In the end, the authors concluded that there are two solutions for node deployment: static and dynamic. In the static method, the proposed algorithm performed better than the other approaches in terms of execution time, and exhibited near-optimal results in the generated coverage sets. The dynamic method on the other hand yielded better results but with a longer execution time.

\subsection{Redundancy Management}

Redundancy plays an important role in maintaining the reliability of a WSN by handling unforeseen failures and boosting the accuracy of calculated computations, due to the infeasibility of replacing the failed sensors. Due to this infeasibility, prolonging the network lifetime to ensure that measurements are taken for as long as possible is another desired trait. The Location-Unaware Coverage (LUC) algorithm [24] addresses the issue of finding a balance between efficient redundancy and network lifetime. The LUC algorithm determines whether a sensory node will be actively receiving measurements or sleeping to preserve its energy. To carry this out, each sensor node determines its neighbors and their relative distance in a two-hop neighborhood. LUC carries out a series of geometric and heuristic tests to determine the 'strength' of the nodes, with the weaker ones put to sleep initially. The stronger nodes possess a higher reliability, and hence are kept active for a longer duration. The LUC algorithm has two protocols: Iterative LUC (LUC-I) and Probabilistic LUC (LUC-P), which determine whether nodes will be set in Active or Sleep mode through a series of hops. The protocols bring about low operating costs and may greatly minimize the number of nodes that are active.

Upon completion of the protocols, all nodes are set to either the Sleep or the Active mode. Active nodes are responsible for covering the entire target area, and the WSN's lifetime and reliability are both improved.

\subsection{Clustering}

The authors in [25] proposed to use the Low-Energy Adaptive Clustering Hierarchy (LEACH) protocol [26] with the Fuzzy Inference System (FIS) based on Mamdani's method [27] to select cluster heads and to extend the lifetime of WSNs. FIS consists of a fuzzifier, an inference engine, and a defuzzifier. The proposed model uses three parameters: distance to coordinator, energy, and density. IF-THEN rules are used to calculate the chance for a sensor node to become a cluster head. The chance value is obtained by defuzzification, which aggregates the results of each rule. Then, the node chooses the maximum of the chance values and compares it to a pre-defined threshold value. If it is found to be less than the threshold then it becomes the cluster head. Experimental studies conducted with LEACH and LEACH-Fuzzy Logic (LEACH-FL) showed that using LEACH-FL increased the network's lifetime twice more than when using LEACH.

\subsection{Set Cover}

A greedy set cover algorithm was proposed by the authors in [28]. It selects the minimum number of sensors that are disseminated into disjoint and non-disjoint sets, with the necessity that every set cover completely fulfills the scope of all its targets. They stated that the proposed algorithm is an improvement over the classical greedy set cover algorithm, and its approximation ratio is verified to be not worse than $\log (m)$. Through simulations, they demonstrated that their algorithm performed better than others from previous research efforts. 


\subsection{Optimization}

Finally, many authors have considered the distribution of sensors and energy optimization as a multi-objective optimization problem. The authors in [29] for instance proposed an Efficient Cover Set Selection (ECSS) approach, which is an improved version of the Non-Dominated Sorting Genetic Algorithm (NSGA-II) [30]. The goal of the proposed scheme is to maintain full coverage by using fewer sensors. They highlighted that having more active sensors results in more coverage but with a lower overall lifetime, and vice versa.

The authors concluded that the ECSS algorithm had advantages such as the ability to reset all the sensors' statuses with a one-time approach. It could also be made flexible by providing it with parameters such as the requested field coverage and the model.

In another approach named the Multi-Objective Set Cover Problem (MO-SCP) [31], the authors considered reliable coverage as an additional conflicting objective to be optimized. They tried to solve it using a multi-objective evolutionary algorithm to select the set with the minimum number of sensor nodes, such that it will provide coverage of all the targets. To meet the requirement of staying active for long periods, the authors proposed to alternate nodes between idle and active states through something called 'wake-up scheduling' of the sensors. Their objective was to achieve a WSN system which can actively sense its predefined targets almost all the time. With the implementation of this method, only a single disjoint set is active while the others are kept in sleep mode (in a specific interval), resulting in an energy conservation scheme. When the active set goes out of power, another set is scheduled to be in the active state. Therefore with this approach, the more DSCs that there are the better it is for the entire system to stay on power. Moreover, they adopted a heuristic crossover operator designed specifically to improve the performance of the algorithm, and conducted simulation scenarios to test the effectiveness of the proposed algorithm.

In this paper, we propose an approach that combines a set cover method with a min-cost max-flow algorithm using a linear programming technique.

\section{Approach}

The proposed algorithm passes through three main phases: (1) information gathering; (2) set cover formation; and (3) path determination.

During the information-gathering phase, all of the sensor nodes including the base station are assigned a unique ID. They then broadcast their IDs using the Carrier-Sense Multiple Access with Collision Avoidance (CSMA/CA) MAC layer protocol, which allows the base station to discover sensor node locations. Now, with the help of the received information about the network, the base station starts the creation of set covers, electing cluster heads and determining optimal routing paths using network layer information. It is to note that once the set cover creation and path determination phases are over, all cluster heads are aware of the next hop to the base station and sensor nodes are also informed about the cluster head ID that they belong to. Then, each cluster head provides a TDMA schedule to its member sensor nodes for intra-cluster communication.

We modeled the energy conservation problem as an optimization problem where a "single objective function" is used for the minimum set covers, and for the min-cost max-flow model too. Our key contribution is that the proposed model guarantees a longer network lifetime.

\subsection{Network Model and Assumptions}

A wireless sensor network is composed of a set of battery-powered sensors and a sink. The latter is connected to a power source and so we disregard its energy consumption in this paper. Communication between the sensors is achieved through wireless links if they are within range of each other, or through other intermediate sensors if not. 
Thus, a WSN can be modeled as a unit disk graph $[32,33] G=(V, E)$ where $V$ is the set of all nodes including the sink and $E \in V \times V$ is a set of possible communication links. We assume our graph is connected.

$S=\left\{s_{1}, s_{2}, \ldots, s_{N}\right\}$ represents a collection of $N$ sensor nodes that are distributed randomly in a two-dimensional plane to monitor a set of target nodes $T=\left\{t_{1}, t_{2}, \ldots ., t_{M}\right\}$.

Given two nodes $s_{1}$ and $s_{2}$ (one of them should be a sensor), they are connected by an edge $\left(s_{1}, s_{2}\right) \in E$, if the first sensor is within the sensing coverage range of the second sensor. Thus, two nodes $s_{1}$ and $s_{2}$ can communicate with each other (there is an edge between them) if ||$d\left(s_{1}, s_{2}\right)|| \leq 1$, where ||$d\left(s_{1}, s_{2}\right)||$ is the Euclidean distance between $s_{1}$ and $s_{2}$.

Each sensor node $s_{i} \in S$, can operate into a number of sensing ranges $r_{1}, r_{2}, \ldots, r_{p}$ where each $r_{k}$ consumes energy $e_{k}, 1 \leq k \leq P$. The initial energy of each sensor node is $E$. A sensing node $s_{i} \in S$ can cover a target $t_{i} \in T$ using sensing range $r_{k}$ if the Euclidean distance $d\left(s_{i}, t_{j}\right)$ is less than or equal to $r_{k}$, denoted as $t_{j} \rightarrow s_{i}$. Then, we define the target subset $T_{i}=\left\{t_{j} \mid t_{j} \rightarrow s_{i}, 1 \leq j \leq m\right\}$ as the target set of the sensor $s_{i}$.

We assume that at regular time intervals, the information sensed by a network node has a fixed unit size of $b$ bits that can be stored at the sensor in a buffer of infinite capacity, modeled as a First In First Out (FIFO) queue. We also assume that the channel bandwidth for all links is $W$ bps and that all transmitted packets have a unit size of $b$ bits. Only one packet can be transmitted in each time slot $t$, which is defined as $t=b / W$ seconds. Time-division multiple access (TDMA) is used at the MAC layer.

\subsection{Cluster Creation}

Our main purpose is to extend the network lifetime as much as possible, and to minimize the energy consumption by keeping the amount of traffic to a minimum and by scheduling nodes to monitor a set of targets continuously in turn. To facilitate the achievement of our objective, a set of clusters is constructed. Many studies have adopted the creation of clusters in WSNs [34-36], however only some of them considered the use of a set cover algorithm. In this study, we consider the construction of a minimum set $\operatorname{cover} C=\{C i\}, 1 \leq i \leq K$ to create clusters. The problem can be described formally as follows.

\section{Set Cover Definition and Creation}

The set cover problem is one of Karp's 21 NP-complete problems shown to be NP-complete in 1972 [37].

The set cover optimization has many applications in different areas [38] including manufacturing, service planning, location problems, network security, and multiple sequence alignments for computational biochemistry [39-42].

Given a set of elements (called the universe) and a collection $S$ of $m$ sets whose union equals the universe, the set cover problem is to identify the smallest sub-collection of $S$ whose union equals the universe.

In our paper we adopt the following approach: using a greedy set cover algorithm [19] to choose which cover a sensor node will belong to. In this approach, we define a finite set $S=\left\{S_{1}, S_{2}, S_{3}, \ldots, S_{m}\right\}$, corresponding to the areas to be monitored, a collection $\left\{S_{j}\right\} n_{j}=1$ of subsets of $S$, where each $S_{j}$ represents a sensor and contains the areas that the sensor monitors from $S$, and a positive integer $k \geq 2$. The goal is to find a partition $G$ of the subsets into $k$ covers $c_{1}, \ldots, c_{k}$ where each cover is a set of subsets, such that $N(G)=k_{i=1}\left|\cup S_{j} \in c_{i} S_{j}\right|$ is minimized.

The heuristic used in our paper consists of greedily picking sets from $S$. The set $S_{j} \in S$ that contains the most number of uncovered vertices is picked at each step. We repeat the process until all vertices are covered. The following is the pseudo-code of the greedy set cover algorithm. 


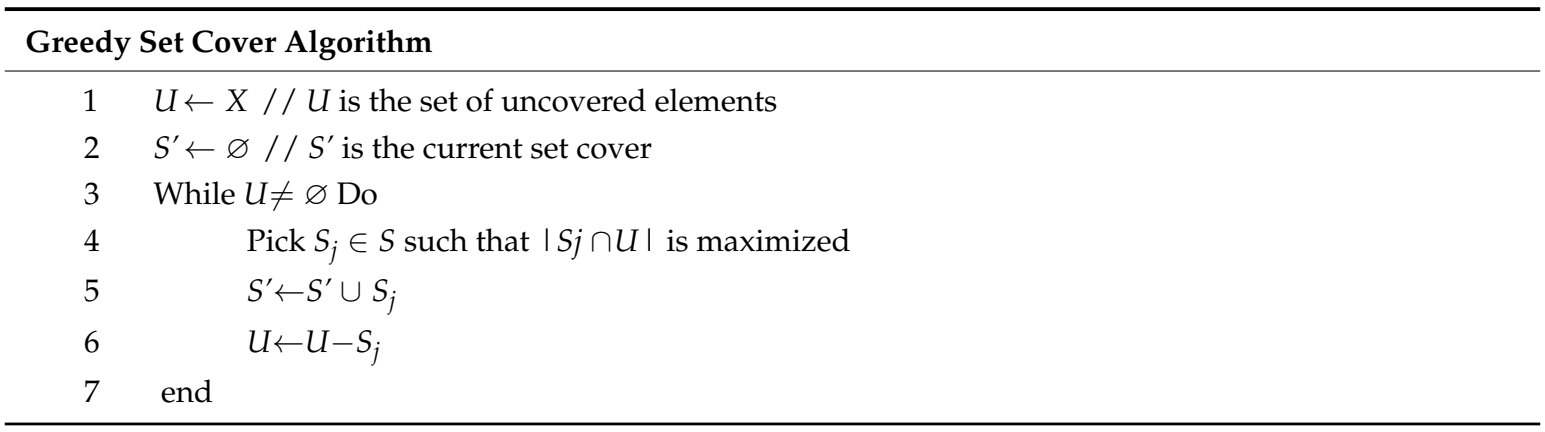

Once the set covers (clusters) are constructed, some sensor nodes become cluster heads (CHs) and collect all traffic from their respective cluster. The cluster head aggregates the collected data and then sends it to the base station as shown in Figure 3. The $\mathrm{CH}$ is chosen based on the highest residual energy, thus allowing the network lifetime to increase in proportion to node density [43].

When using clustering, the workload on the cluster head is thus larger than for non-cluster heads. To maximize the lifetime of cluster heads, we propose to elect two cluster heads within each cluster that have the highest energy, and interchange their roles in a round-robin fashion during their lifetime in order to distribute and balance the extra workload and energy consumption evenly between them.

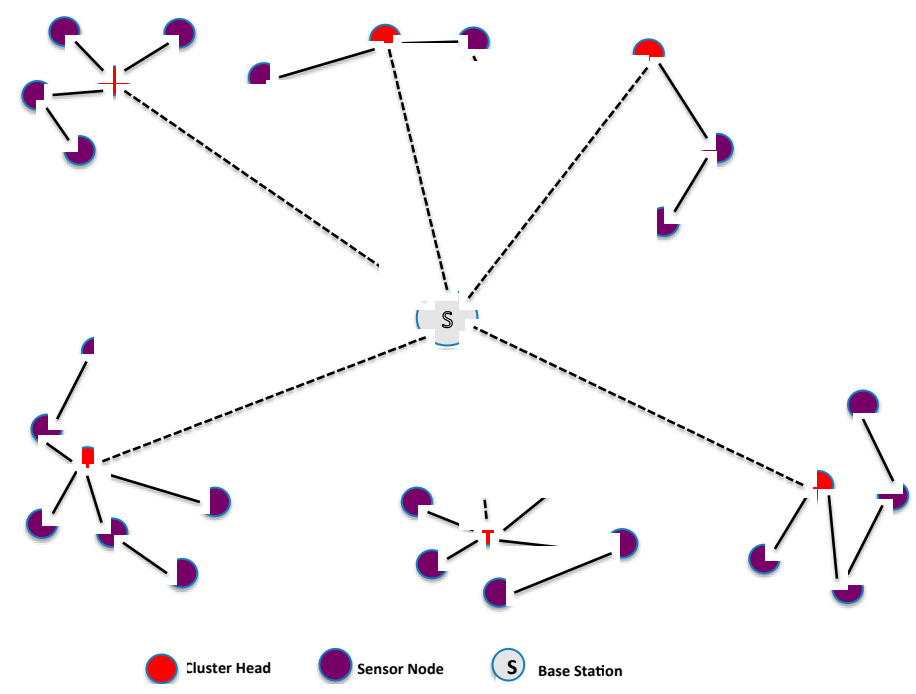

Figure 3. Cluster creation with cluster head elected.

\subsection{Energy Consumption Model}

The energy consumption model adopted in this paper is based on the proposed model in [44] which has been used by many researchers [45-50]. In this model, it is assumed that the transmitter, power amplifier, and receiver all dissipate energy to run the radio electronics as shown in Figure 4.

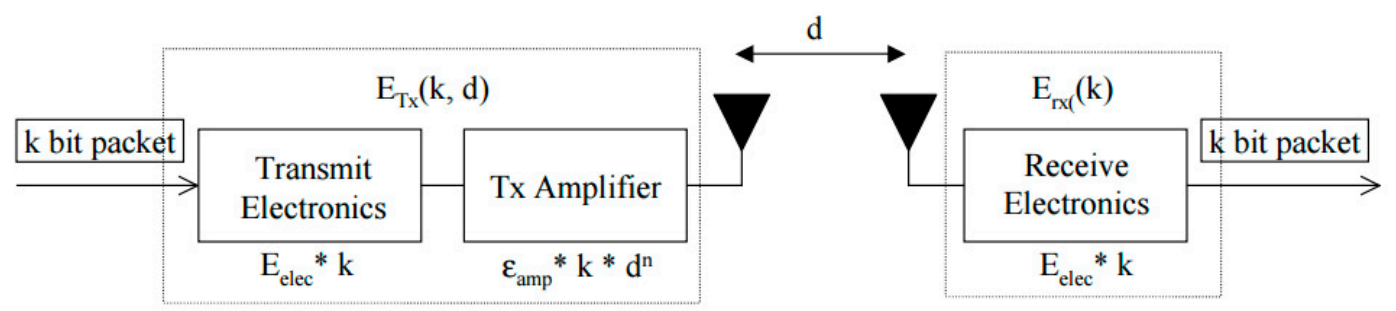

Figure 4. Radio Energy Dissipation Model. 
The power attenuation is dependent on the distance between the transmitter and the receiver. For relatively short distances, the propagation loss can be modeled as inversely proportional to $d^{2}$, whereas for longer distances the propagation loss can be modeled as inversely proportional to $d^{4}$. Power control can be used to invert this loss by setting the power amplifier to ensure a certain power at the receiver. Thus, to calculate the transmission cost (joule/bit) and the receiving cost (joule/bit) for a message of size $k$ bits from a sensor node $i$ (transmitter: $T_{x}$ ) to a sensor node $j$ (receiver: $R_{x}$ ) over a distance $d(i, j)$ (Euclidian-Distance), where the energy consumption at node $i$ comprises operating radio electronics and amplifiers while at node $j$ only radio electronics, the following equations are used:

Transmission cost:

$$
E_{T X}(k, d)=k \times E_{T X-e l e c}+E_{T X-a m p}(k, d)=k \times E_{T X \text {-elec }}+k \times d^{2} \times E_{a m p},
$$

Receiving cost:

$$
E_{R X}(k, d)=k \times E_{R X-e l e c},
$$

where $E_{\text {elec }}$ is the energy dissipation of the radio in order to run the transmitter and receiver circuitry, and $E_{\text {amp }}$ is the transmitter amplifier.

Typical values used in this paper during the simulation are:

$E_{\text {elec }}=50 \mathrm{~nJ} / \mathrm{bit}, E_{\text {amp }}=100 \mathrm{pJ} / \mathrm{bit} / \mathrm{m}^{2}, d^{2}=500$, and the packet size $k=2000$ bits [49].

If we consider that node $i$ wants to transmit an amount of data $f_{i j}$ to node $j$, the energy consumption (Joules) at node $i$ will be:

$$
E_{T X, I}=\sum_{\substack{j=1 \\ i \neq j}}^{n}\left(E_{T X, i j} * f_{i j}\right)
$$

On the other hand, if node $j$ wants to send an amount of data $f_{j i}$ to node $i$, the energy consumption (Joules) at node $i$ will be:

$$
E_{R X, I}=E_{R X} \sum_{\substack{j=1 \\ i \neq j}}^{n} f_{j i}
$$

Thus, the total energy consumed at node $i$ will be:

$$
E_{\text {consu }, i}=E_{T X, i}+E_{R X, I}
$$

\subsection{Energy Optimization}

Once the clusters are created, our objective is to minimize the energy consumption when routing data to the base station. First, we minimize energy consumption within each cluster, and then we minimize the routing energy consumption.

\subsubsection{Minimizing Energy Consumption in Clusters}

The objective of using a SET K-COVER approach to construct clusters in WSNs is to increase their energy efficiency. The reason for the common placement of multiple sensors close together to cover a single area is due to the ad-hoc nature of sensor placement, topological constraints, or to compensate for the short lifetime of the sensors.

Therefore, in an effort to increase the longevity of the network and to conserve battery power, it is beneficial to activate groups of sensors in rounds, so that the battery life of a sensor is not wasted on areas that are already monitored by other sensors. Additionally, certain batteries last up to twice as long when used in short bursts as opposed to continuously. Therefore, activating a sensor only once every $k$ time units can extend the lifetime of its battery. For the active nodes inside a cluster, an energy cost $e_{i}$ is associated with a sensor node $x_{i}$ each time the latter sends a message to the $\mathrm{CH}$. 
Thus, the energy consumption for a cluster can be formulated as a linear programming optimization problem where:

$$
\text { Minimizing } \sum_{i=1}^{k} x_{i} * e_{i},
$$

where $k$ is the number of active nodes inside the cluster.

St:

$$
\begin{gathered}
e_{i} \leq E_{\text {init }} \\
x_{i}= \begin{cases}1 & \text { if active } \\
0 & \text { if inactive }\end{cases} \\
\sum_{1}^{k} e_{i} \leq E_{\text {clust }}
\end{gathered}
$$

where $E_{\text {init }}$ is the initial energy of node $x_{i}$, where $E_{\text {clust }}$ is the initial cluster total energy of all nodes.

Output: vector $X=\left(x_{1}, x_{2}, \ldots x_{n}\right)$ for nodes active and inactive.

\subsubsection{Routing Energy Optimization}

It is to note that in order to achieve the effective collection of data, WSNs must meet two requirements. One is full coverage of the targets, and the other is complete connectivity of the network. Full coverage means that the sensors should be able to monitor all targets, and complete connectivity means that the data that originated from the monitoring targets should be able to reach the base station through multi-hop wireless communication using a minimum energy path.

We believe that relying only on a minimum energy routing strategy has some deficiency in terms of network lifetime. Since most of the time using a relay node is more energy-efficient than direct communication with the base station, sensor nodes closer to the base station exhaust their batteries long before other nodes residing on the perimeter of the network area. Hence, network lifetime cannot be optimized with minimum energy routing alone. As a solution, we propose to take into consideration the residual energy of the nodes in the path by assigning link costs inversely proportional to the residual energy values. This way, energy consumption could be balanced throughout the whole network. In fact, such a solution can achieve maximal network lifetime using a linear programming (LP) based model.

\section{Network Model}

After clustering, the routing procedure is invoked during data transmission using a min-cost max-flow algorithm [51].

The Wireless Sensor Network used in this paper is modeled as a directed graph $G(V, E)$, where $V$ represents the set of sensor nodes and $E$ the set of edges.

Each edge $(u, v) \in E$ is associated with a cost $c(u, v)$ which represents the energy consumption when there is a flow $f_{i j}$ going out from $u$ to $v$, a capacity constraint $u_{i j}$ expressed in the number of packets per unit time, and a supplies/demands variable $d_{i j}$. The cost of a flow is: $f(u, v) . c(u, v)$.

The objective is to deliver all the data generated by the sensor nodes to the base station with minimum energy consumption and without exceeding the link capacities.

The problem can be formulated as follows:

Minimizing the total cost of the flow over all edges:

$$
\sum_{(u, v) \in E} f(u, v) \cdot c(u, v)
$$

St:

$$
\begin{gathered}
f(u, v) \leq c(u, v) \forall(u, v) \in E \\
f(u, v)=-f(v, u)
\end{gathered}
$$




$$
\begin{aligned}
& \sum_{w \in V} f(u, v)=0 \text { for all } u \neq s, t \\
& \sum_{w \in V} f(v, w)-\sum_{w \in V} f(w, v)=d
\end{aligned}
$$

where (11) represents the capacity constraint, (12) the skew symmetry, (13) the flow conservation, and (14) the required flow.

The above problem can be converted into a minimum-cost circulation problem [52] and can be solved efficiently using well-known min-cost flow algorithms [53-56]. In this research we adopt a network simplex method [57] as it is considered the most practical algorithm [57]. Figure 5 shows a sample of a sensor network where $s_{1}$ is the source and $s_{6}$ is the destination. The flow capacities and demands are shown on the graph.

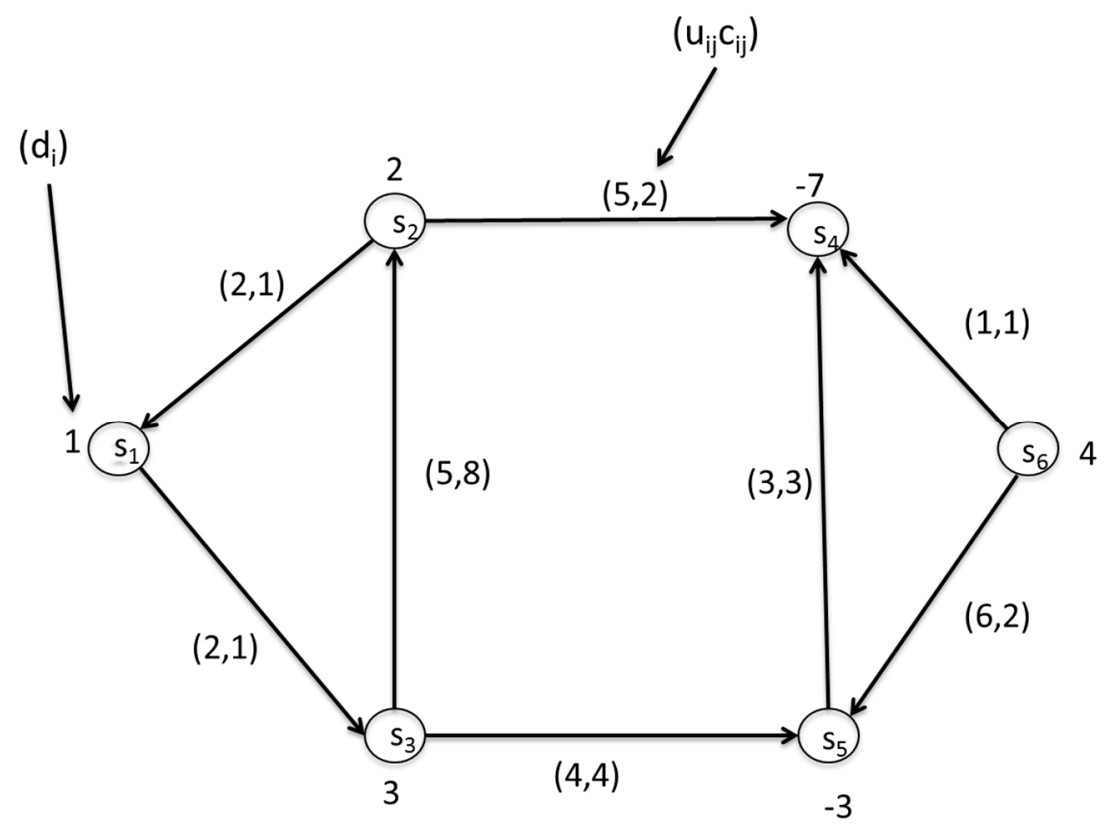

Figure 5. WSN graph representation.

The corresponding network simplex LP is as follows, where $x_{i j}=$ Number of units shipped from node $i$ to $j$ using arc $i-j$ :

$$
\begin{gathered}
\text { Min } z=x_{13}+2 x_{21}+2 x_{24}+8 x_{32}+4 x_{35}+3 x_{54}+x_{64}+2 x_{65} \\
\text { Node } s_{1}: x_{13}-x_{21}=1 \\
\text { Node } s_{2}: x_{21}+x_{24}-x_{32}=2 \\
\text { Node } s_{3}:-x_{13}+x_{32}+x_{35}=3 \\
\text { Node } s_{4}:-x_{24}-x_{54}-x_{64}=-7 \\
\text { Node } s_{5}:-x_{35}+x_{54}-x_{65}=-3 \\
\text { Node } s_{6}: x_{64}+x_{65}=4 \\
x_{13} \leq 2 x_{21} \leq 2 x_{24} \leq 5 x_{32} \leq 5 x_{35} \leq 4 x_{54} \leq 3 x_{64} \leq 1 x_{65} \leq 6
\end{gathered}
$$

\section{Simulation Results}

In order to assess our model, we compared it to the MO-SCP, MDP-MSC, and LEACH models.

We performed a set of simulation scenarios in MATLAB [58] using a variable number of sensor nodes $(100,200,300,400)$ deployed randomly in a $1500 \mathrm{~m}^{2}$ area. This allowed us to evaluate the 
effectiveness of our proposed approach and analyze its impact on the energy of the entire network, and thus on the lifetime of the WSN. Parameters used in the simulation are similar to those used in [59], and are shown in Table 1.

Table 1. Simulation parameters.

\begin{tabular}{cc}
\hline Parameters & Value \\
\hline Initial energy $E_{\text {init }}$ & $0.5 \mathrm{~J}$ \\
Transmitting and receiving energy $E_{\text {elec }}$ & $5 \mathrm{~nJ} / \mathrm{bit}$ \\
Amplification energy for short distance $E_{f}$ & $10 \mathrm{Pj} / \mathrm{bit} / \mathrm{m}^{2}$ \\
Amplification energy for long distance $E_{a m p}$ & $0.013 \mathrm{pJ} / \mathrm{bit} / \mathrm{m}^{4}$ \\
Packet size & $200 \mathrm{bits}$ \\
Operating frequency & $2.4 \mathrm{GHz}$ \\
Initial energy of sensor & $61,560 \mathrm{~J}$ \\
Transmit power & $32 \mathrm{~mW}$ \\
Reference distance & $3 \mathrm{~m}$ \\
Path loss exponent & 4 \\
Signal to Noise Ratio & $2 \mathrm{~dB}$ \\
\hline
\end{tabular}

Our experiment consists of two phases of WSN network design: cluster set cover creation and path determination.

In both phases, we input the set of SNs, their locations, the required network lifetime, and other parameters to the corresponding SCMC model. We then solved the WSN design model with the IBM ${ }^{\circledR}$ ILOG $^{\circledR}$ OPL-CPLEX ${ }^{\circledR}$ optimization solver.

In the first phase the set cover model is used to obtain the optimal number of set covers with their corresponding $\mathrm{CH}$, and the optimal packet transmission path from $\mathrm{SNs}$ to the selected $\mathrm{CH}$ inside each cluster. In the second phase we use the results obtained from the first phase to derive the optimal path between the $\mathrm{CH}$ and the $\mathrm{BS}$ using the min-cost max-flow algorithm. The metrics used to test the effectiveness of our proposed approach are based on the percentage of Alive nodes, the round where the First Node Dies (FND), the round where the Last Node Dies (LND), and the network throughput.

The results of the first phase are shown in Table 2. The Greedy algorithm creates a number of set covers for each scenario where the number of sensor nodes is varied $(N=100,200,300$ and 400$)$

Table 2. Set covers number created by Greedy algorithm.

\begin{tabular}{cc}
\hline Number of Nodes & Number of Clusters \\
\hline 100 & 7 \\
200 & 8 \\
300 & 7 \\
400 & 8 \\
\hline
\end{tabular}

In the second phase, the min-cost max-flow model is solved as stated before and a comparison is carried out between the different approaches on the basis of the metrics stated earlier. Tables 3 and 4 summarize the results of these metrics.

Table 3. Number of rounds when $10 \%, 20 \%, 30 \%, 40 \%, 50 \%, 60 \%, 70 \%$ and $80 \%$ of nodes die.

\begin{tabular}{ccccccccc}
\hline \multirow{2}{*}{ Protocol } & \multicolumn{8}{c}{ Percentage of Alive Nodes } \\
\cline { 2 - 9 } & $\mathbf{9 0}$ & $\mathbf{8 0}$ & $\mathbf{7 0}$ & $\mathbf{6 0}$ & $\mathbf{5 0}$ & $\mathbf{4 0}$ & $\mathbf{3 0}$ & $\mathbf{2 0}$ \\
\hline LEACH & 1100 & 1642 & 1600 & 1465 & 2278 & 2500 & 2986 & 2987 \\
MDP-MSC & 1600 & 1855 & 1900 & 1978 & 2436 & 2875 & 3100 & 3300 \\
MO-SCP & 1756 & 1860 & 2150 & 2360 & 2780 & 3164 & 3600 & 3785 \\
SCMC & 1865 & 1890 & 2260 & 2600 & 2830 & 3700 & 4300 & 4500 \\
\hline
\end{tabular}


Table 4. Nodes die-off statistics of the different approaches.

\begin{tabular}{ccc}
\hline Protocol & FND & LND \\
\hline LEACH & 1019 & 1986 \\
MDP-MSC & 1125 & 3125 \\
MO-SCP & 1019 & 4035 \\
SCMC & 975 & 9800 \\
\hline
\end{tabular}

Table 3 shows the number of rounds completed by various schemes when $10 \%, 20 \%, 30 \%, 40 \%$, $50 \%, 60 \%, 70 \%$ and $80 \%$ of nodes die for $N=100$.

It is evident, as shown in Table 3, that SCMC has a better performance than its counterpart protocols. For instance, in the case of SCMC the number of rounds when $80 \%$ of nodes are dead is 4500 whereas in the case of MO-SCP, MDP-MSC, and LEACH the number is 3785, 3300, and 2987 respectively. Therefore, SCMC extends the lifetime of the WSN and can provide information for a considerably longer period of time. This lifetime increases with node density, where SCMC takes the advantage of the set cover formation and the optimal routing energy.

Despite the fact that SCMC has its first node dead in an earlier round than the other approaches, it shows very promising results, with the death of all nodes as shown in Table 4 occurring at about 9800 rounds compared to 4035 for MO-SCP, 3125 for MDP-MSC, and 1986 for LEACH. Furthermore, we clearly see that when $20 \%$ of nodes at the same number of rounds in SCMC are alive, all nodes in the other approaches are dead.

In the second experiment we calculated the throughput for all schemes with a different variation of sensor nodes from 100 to 400 with an increment of 100. We observe as shown in Figures 6-9 that SCMC outperforms other algorithms in terms of a higher throughput and a larger number of packets received at the base station, which is understandable as SCMC has a longer lifetime and it uses an optimized routing path compared to other algorithms.

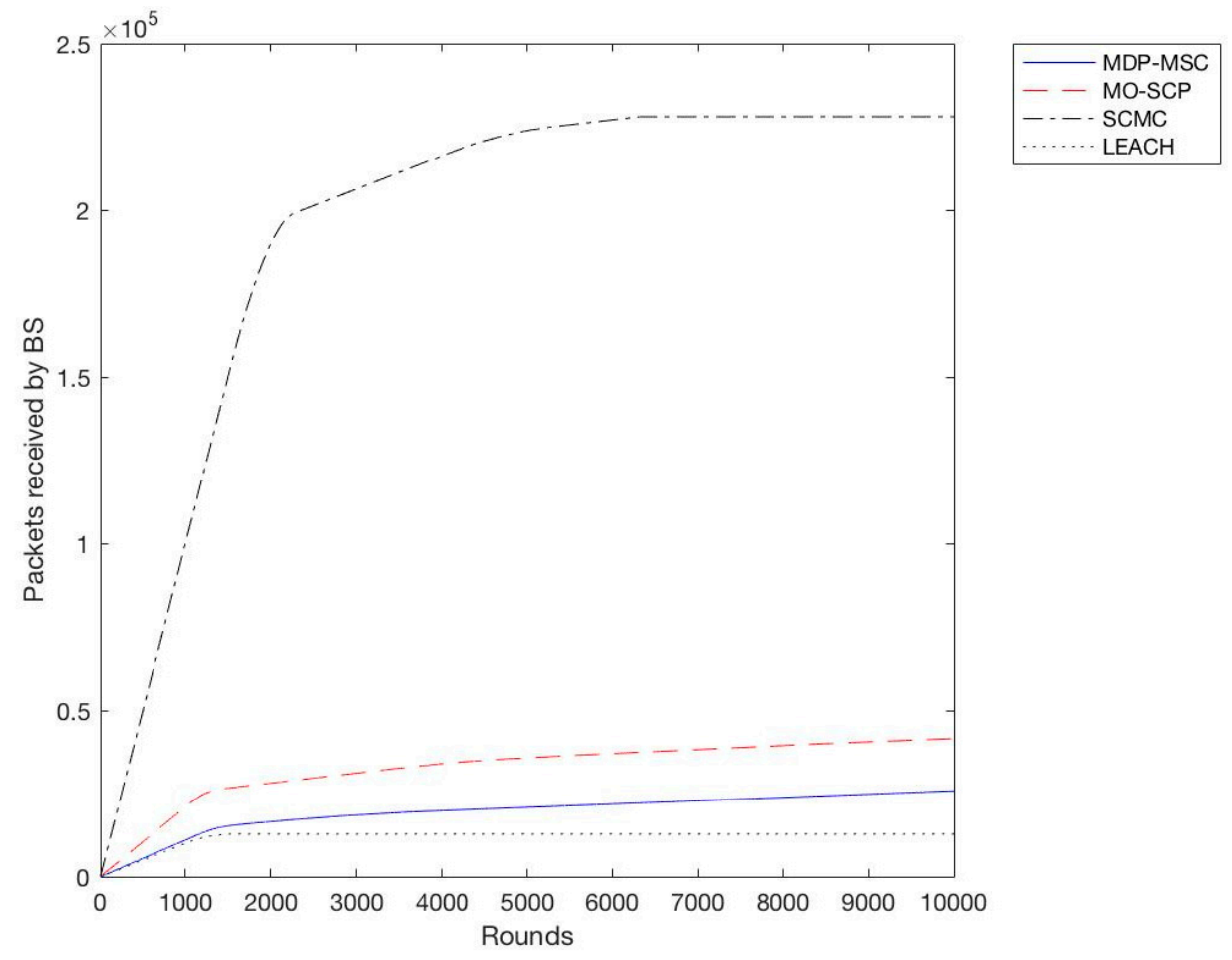

Figure 6. Throughput comparison of Set Cover Min-Cost max-flow (SCMC), Multi-Objective Set Cover Problem (MO-SCP), Minimum Distance Path-Maximum Set Cover (MDP-MSC), and Low-Energy Adaptive Clustering Hierarchy $(\mathrm{LEACH})(\mathrm{Size}=100)$. 
Based on the previous simulation results, we have shown that SCMC provides a higher throughput and a prolonged lifetime of the WSN compared to MO-SCP, MDP-MSC, and LEACH. This is due to the fact that SCMC uses a set cover approach to create clusters, and min-cost max-flow to route data between $\mathrm{CHs}$ and the base station. Although MDP-MSC also uses the concept of set covers to create clusters, it remains different from MO-SCP and SCMC in three ways. Firstly, it searches for $\mathrm{k}$ paths from the sink to all sensor nodes while MO-SCP and SCMC use a single path approach. Secondly, it calculates maximum disjoint set covers while MO-SCP and SCMC calculate a minimum set cover. Furthermore, MDP-MSC doesn't consider the problem of extending the lifetime of WSNs as an optimization problem, while MO-SCP considers it as a multi-objective optimization problem, and SCMC as a single objective problem. In SCMC the energy consumption is well distributed among nodes and routing is done in an efficient way.

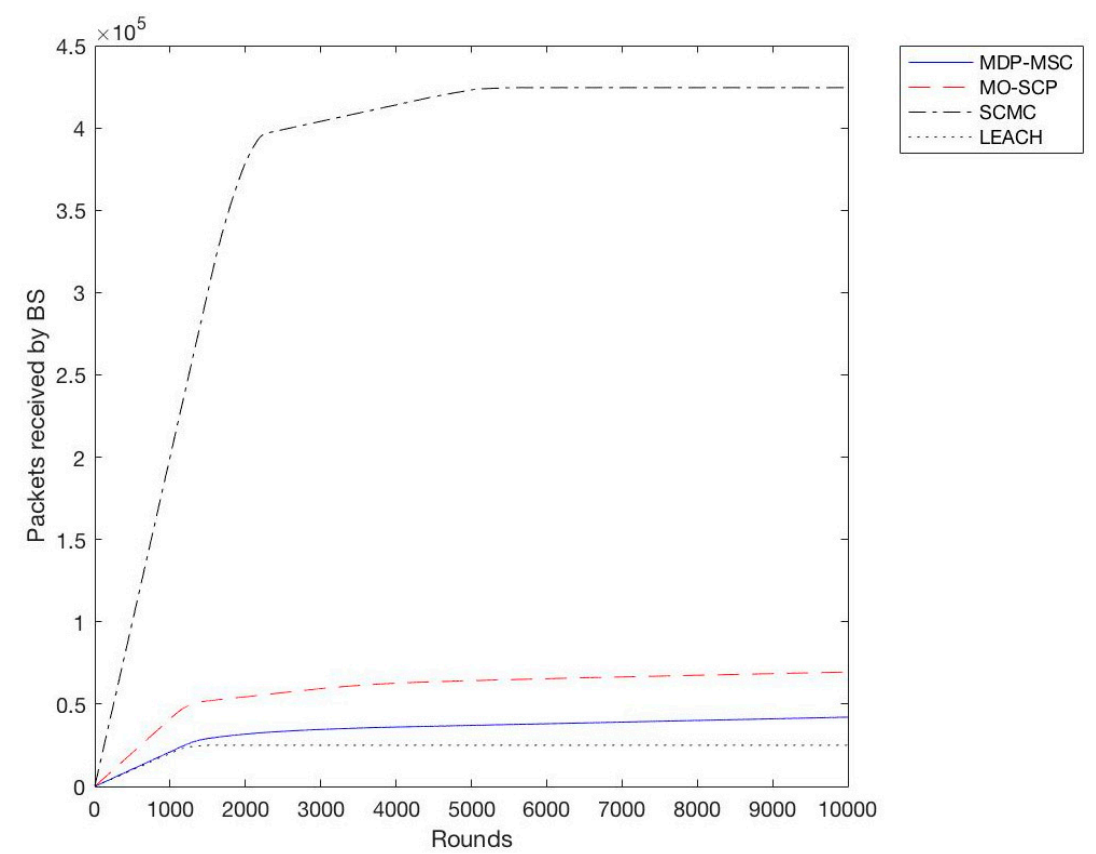

Figure 7. Throughput comparison of SCMC, MO-SCP, MDP-MSC and LEACH (Size = 200).

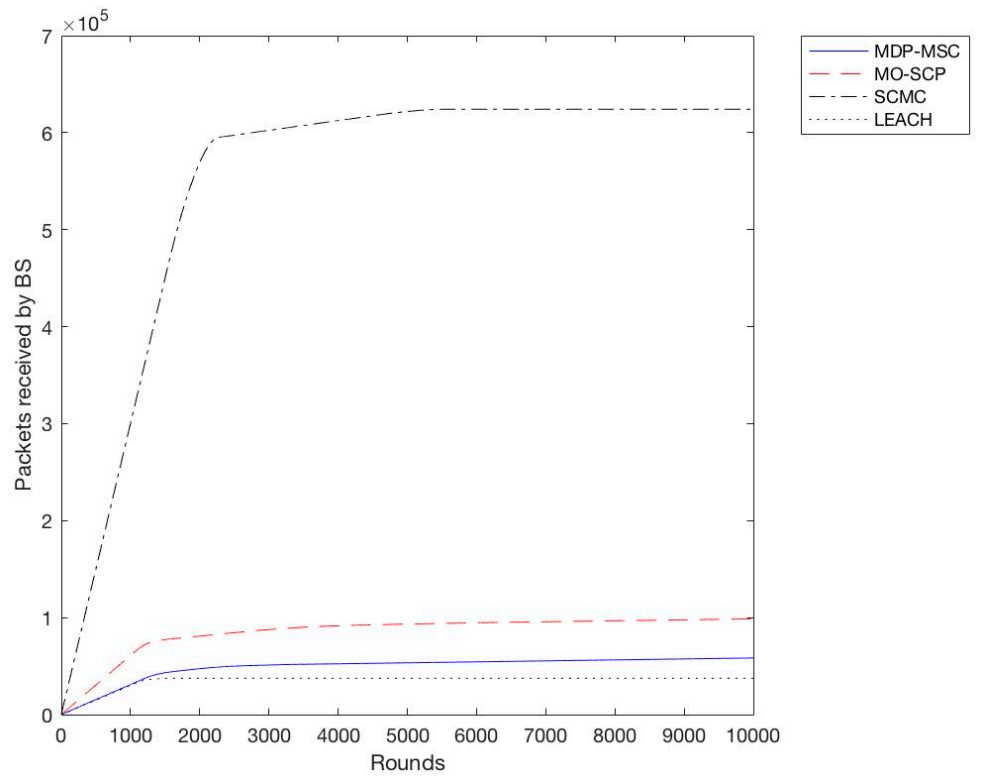

Figure 8. Throughput comparison of SCMC, MO-SCP, MDP-MSC and LEACH (Size = 300). 


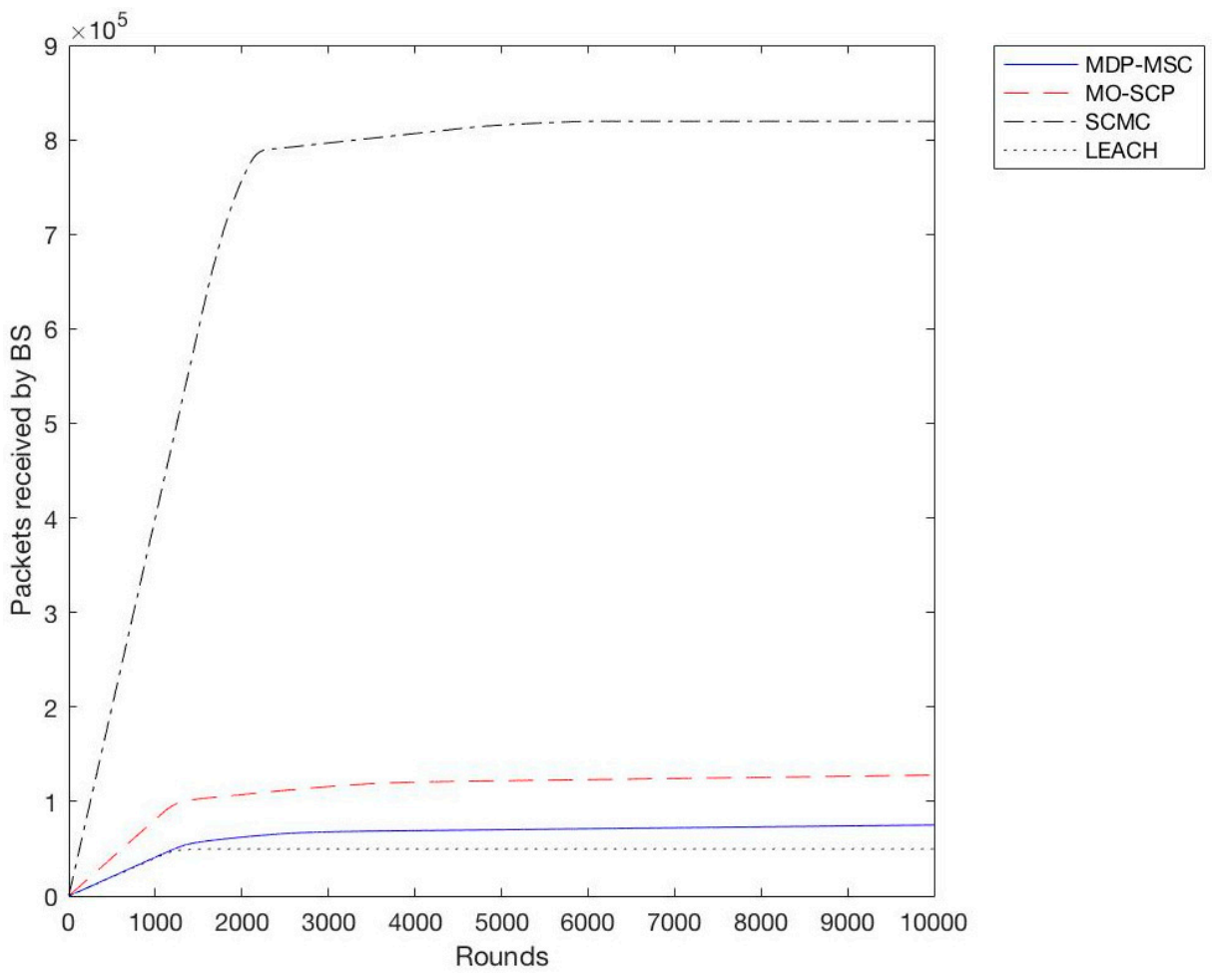

Figure 9. Throughput comparison of SCMC, MO-SCP, MDP-MSC and LEACH (Size = 400).

\section{Conclusions}

In this paper, we extensively studied the problem of minimizing the energy consumption and maximizing the lifetime of WSNs. For this purpose, an effective approach was presented using an optimization model. The proposed approach controls the energy depletion of sensor nodes and minimizes the routing path using the residual energy of the nodes. The corresponding optimization objective functions were solved using linear programing, and the simulation results showed that the proposed scheme (SCMC) outperforms MO-SCP, MDP-MSC, and LEACH in terms of WSN lifetime and throughput. As a future project, we intend to apply our approach to various base station scenarios. Additionally, the use of a multi-objective optimization model would be of great interest.

Acknowledgments: Project funded by Research Translation Center (RTC), Grant No. GP-CCIS-2013-11-17.

Conflicts of Interest: The author declares no conflict of interest. The founding sponsors had no role in the design of the study; in the collection, analyses, or interpretation of data; in the writing of the manuscript, and in the decision to publish the results.

\section{References}

1. Rohankar, R.; Katti, C.P.; Kumar, S. Comparison of Energy Efficient Data Collection Techniques in Wireless Sensor Network. Procedia Comput. Sci. 2015, 57, 146-151. [CrossRef]

2. More, A.; Raisinghani, V. A survey on energy efficient coverage protocols in wireless sensor networks. J. King Saud Univ. Comput. Inf. Sci. 2017, 29, 428-448. [CrossRef]

3. Touati, Y.; Ali-Chérif, A.; Daachi, B. Routing Information for Energy Management in WSNs. In Energy Management in Wireless Sensor Networks; Elsevier: Amsterdam, The Netherlands, 2017; pp. 23-51. [CrossRef]

4. Wang, K.; Ou, Y.; Ji, H.; Zhang, H.; Li, X. Energy aware hierarchical cluster-based routing protocol for WSNs. J. China Univ. Posts Telecommun. 2016, 23, 46-52. [CrossRef]

5. Elhoseny, M.; Tharwat, A.; Yuan, X.; Hassanien, A.E. Optimizing K-coverage of mobile WSNs. Expert Syst. Appl. 2018, 92, 142-153. [CrossRef] 
6. Naeem, M.; Iqbal, M.; Anpalagan, A.; Ahmad, A.; Obaidat, M.S. Chapter 15-Optimization classification and techniques of WSNs in smart grid. In Smart Cities and Homes; Obaidat, M., Nicopolitidis, P., Eds.; Morgan Kaufmann: Burlington, MA, USA, 2016; pp. 323-343. [CrossRef]

7. Kuila, P.; Jana, P.K. Energy efficient clustering and routing algorithms for wireless sensor networks: Particle swarm optimization approach. Eng. Appl. Artif. Intell. 2014, 33, 127-140. [CrossRef]

8. Singh, B.; Lobiyal, D.K. Energy-aware Cluster Head Selection Using Particle Swarm Optimization and Analysis of Packet Retransmissions in WSN. Procedia Technol. 2012, 4, 171-176. [CrossRef]

9. Obaidy, M.A.; Ayesh, A. Energy efficient algorithm for swarmed sensors networks. Sustain. Comput. Inf. Syst. 2015, 5, 54-63. [CrossRef]

10. Azharuddin, M.; Jana, P.K. Particle swarm optimization for maximizing lifetime of wireless sensor networks. Comput. Electr. Eng. 2016, 51, 26-42. [CrossRef]

11. Mortazavi, S.H.; Salehe, M.; MacGregor, M.H. Maximum WSN coverage in environments of heterogeneous path loss. Int. J. Sens. Netw. 2014, 16, 185-198. [CrossRef]

12. Peng, J.; Chen, Y. A low energy consumption WSN node. Int. J. Embed. Syst. 2015, 7, 318-323. [CrossRef]

13. Zhou, Y.; Wang, X.; Wang, T.; Liu, B.; Sun, W. Fault-tolerant multi-path routing protocol for WSN based on HEED. Int. J. Sens. Netw. 2016, 20, 37-45. [CrossRef]

14. Gupta, S.; Roy, K.C. Q-Coverage Maximum Connected Set Cover (QC-MCSC) Heuristic for Connected Target Problem in Wireless Sensor Network. Glob. J. Comput. Sci. Technol. 2015, 15, 470-474.

15. Jain, S.; Gupta, S. Analysis and Comparison of Wireless Sensor Networks Coverage Algorithms. Int. J. Comput. Sci. Inf. Technol. 2014, 5, 1668-1670.

16. Attea, B.A.; Khalil, E.A.; Ozdemir, S.; Yıldız, O. A multi-objective disjoint set covers for reliable lifetime maximization of wireless sensor networks. Wirel. Pers. Commun. 2015, 81, 819-838. [CrossRef]

17. Jia, J.; Chena, J.; Chang, G.; Wena, Y.; Song, J. Multi-objective optimization for coverage control in wireless sensor network with adjustable sensing radius. Comput. Math. Appl. 2009, 57, 1767-1775. [CrossRef]

18. Ji, S.; Pan, Y.; Li, Y. Reliable and Energy Efficient Target Coverage for Wireless Sensor Networks. Tsinghua Sci. Technol. 2011, 16, 464-474.

19. Diop, B.; Diongue, D.; Thiaré, O. Greedy Algorithms for Target Coverage Lifetime Management Problem in Wireless Sensor Networks. Int. J. Control Autom. 2015, 8, 232-250. [CrossRef]

20. Younis, O.; Krunz, M.; Ramasubramanian, S. Location-Unaware coverage in wireless sensor networks. Ad Hoc Netw. 2008, 6, 1078-1097. [CrossRef]

21. Yao, L.; Zeng, F. Maximum Set Covers Based Energy Conservation Scheme in Wireless Sensor Networks. Tech. Gaz. 2014, 21, 733-739.

22. Cardei, M.; Thai, M.T.; Li, Y.; Wu, W. Energy-efficient target coverage in wireless sensor networks. In Proceedings of the 24th Annual Joint Conference of the IEEE Computer and Communications Societies (INFOCOM 2005), Miami, FL, USA, 13-17 March 2005; IEEE: Piscataway, NJ, USA.

23. Liu, L.; Hu, B.; Miao, H.; Li, H.; Li, L.; Zhao, Q. Achieving Energy Conservation, Coverage and Connectivity Requirements in Wireless Sensor Networks. In Proceedings of the 29th IEEE International Conference on Distributed Computing Systems Workshops, Montreal, QC, Canada, 22-26 June 2009.

24. Idrees, A.K.; Deschinkel, K.; Salomon, M.; Couturier, R. Distributed lifetime coverage optimization protocol in wireless sensor networks. J. Supercomput. 2015, 71, 4578-4593. [CrossRef]

25. Belghith, O.B.; Sbita, L. Extending the network lifetime of wireless sensor networks using fuzzy logic. In Proceedings of the IEEE 12th International Multi-Conference on Systems, Signals \& Devices, Mahdia, Tunisia, 16-19 March 2015.

26. Handy, M.J.; Haase, M.; Timmermann, D. Low energy adaptive clustering hierarchy with deterministic cluster-head selection. In Proceedings of the 4th International Workshop on Mobile and Wireless Communications Network, Stockholm, Sweden, 9-11 September 2002; pp. 368-372.

27. Mamdani, E.H.; Assilian, S. An experiment in linguistic synthesis with a fuzzy logic controller. Int. J. Man-Mach. Stud. 1975, 7, 1-13. [CrossRef]

28. Zorbas, D.; Glynos, D.; Kotzanikolaou, P.; Douligeris, C. Solving coverage problems in wireless sensor networks using cover sets. Ad Hoc Netw. 2010, 8, 400-415. [CrossRef]

29. Jia, J.; Chen, J.; Chang, G.-R.; Wen, Y.-Y. Efficient cover set selection in wireless sensor networks. Acta Autom. Sin. 2008, 34, 1157-1162. [CrossRef] 
30. Deb, K.; Pratap, A.; Agarwal, S.; Meyarivan, T. A Fast and Elitist Multiobjective Genetic Algorithm: NSGA-II. IEEE Trans. Evol. Comput. 2002, 6, 182-197. [CrossRef]

31. Attea, B.A. Multi-Objective Set Cover Problem for Reliable and Efficient Wireless Sensor Networks. Iraqi J. Sci. 2015, 56, 1147-1160.

32. Clark, B.N.; Colbourn, C.J.; Johnson, D.S. Unit disk graphs. Discret. Math. 1990, 86, 165-177. [CrossRef]

33. Purohit, G.N.; Verma, S.; Sharma, U. Powers of a Graph and Associated Graph Labeling. Int. J. Comput. Netw. Secur. 2010, 2, 45-49.

34. Jia, J.; Chen, J.; Chang, G.; Li, J. Find the maximum k-disjoint coverage sets in WSN using genetic algorithm. Int. J. Model. Identif. Control 2010, 9, 43-52. [CrossRef]

35. Prabakaran, T.; Karthikeyan, N.K. Adaptive clustering and routing using fuzzy decision model in WSN. Int. J. Mob. Netw. Des. Innov. 2015, 6, 51-62. [CrossRef]

36. Ali, N.A.; ElSayed, H.M.; El-Soudani, M.; Amer, H.H.; Daoud, R.M. Elongation of WSN lifetime using a centralised clustering technique. Int. J. Syst. Control Commun. 2012, 4, 250-261. [CrossRef]

37. Karp, R.M. Reducibility among Combinatorial Problems. In Complexity of Computer Computations; Miller, R.E., Thatcher, J.W., Eds.; Plenum: New York, NY, USA, 1972; pp. 85-103.

38. Gomes, F.C.; Meneses, C.N.; Pardalos, P.M.; Viana, G.V.R. Experimental analysis of approximation algorithms for the vertex cover and set covering problems. Comput. Oper. Res. 2006, 33, 3520-3534. [CrossRef]

39. Lan, G.; DePuy, G.W.; Whitehouse, G.E. An effctive and simple heuristic for the set covering problem. Europ. J. Oper. Res. 2007, 176, 1387-1403. [CrossRef]

40. Ruan, L.; Du, H.; Jia, X.; Wu, W.; Li, Y.; Ko, K.I. A greedy approximation for minimum connected dominating sets. Theor. Comput. Sci. 2004, 32, 325-330. [CrossRef]

41. Bilal, N.; Galinier, P.; Guibault, F. A New Formulation of the Set Covering Problem for Metaheuristic Approaches. Int. Sch. Res. Not. 2013, 1-10. [CrossRef]

42. Akhter, F. A Heuristic Approach for Minimum Set Cover Problem. Int. J. Adv. Res. Artif. Intell. 2015, 4, 40-45. [CrossRef]

43. Xu, Y.; Heidemann, J.; Estrin, D. Geography-informed energy conservation for Ad Hoc routing. In Proceedings of the 7th Annual International Conference on Mobile Computing and Networking, Rome, Italy, 16-21 July 2001; pp. 70-84.

44. Heinzelman, W. Application Specific Protocol Architectures for Wireless Networks. Ph.D. Thesis, Massachusetts Institute of Technology (MIT), Cambridge, MA, USA, 2000.

45. Prajapat, M.; Barwar, N.C. Performance Analysis of Energy Dissipation in WSNs Using Multi-Chain PEGASIS. Int. J. Comput. Sci. Inf. Technol. 2014, 5, 8033-8036.

46. Acharya, A.; Seetharam, A.; Bhattacharyya, A.; Naskar, M.K. Balancing energy dissipation in data gathering wireless sensor networks using ant colony optimization. In Proceedings of the International Conference on Distributed Computing and Networking, Hyderabad, India, 3-6 January 2009; pp. 437-443.

47. Lindsey, S.; Raghavendra, C.S. PEGASIS: Power Efficient Gathering in Sensor Information Systems. In Proceedings of the IEEE ICC, Big Sky, MT, USA, 9-16 March 2001; pp. 1125-1130.

48. Tan, H.O.; Korpeoglu, I. Power efficient data gathering and aggregation in wireless sensor networks. ACM SIGMOD Rec. 2003, 32, 66-71. [CrossRef]

49. How Can I Start for Calculating Energy Dissipation in WSN? ResearchGate. Available online: https: / / www.researchgate.net/post/How_can_I_start_for_calculating_energy_dissipation_in_WSN (accessed on 9 December 2017).

50. Heinzelman, W.; Chandrakasan, A.; Balakrishna, H. Energy-Efficient Communication Protocol for Wireless Microsensor Networks. In Proceedings of the 33rd Hawaii International Conference on System Sciences, Maui, HI, USA, 7 January 2000; pp. 1-10.

51. Ahuja, R.; Magnanti, T.; Orlin, J. Network Flows; Prentice Hall: Upper Saddle River, NJ, USA, 1993.

52. Goldberg, A.V.; Tarjan, R.E. Solving minimum-cost flow problems by successive approximation. In Proceedings of the Nineteenth Annual ACM Symposium on Theory of Computing, New York, NY, USA, 25-27 May 1987.

53. Bixby, R.E.; Wagner, D.K. An almost linear-time algorithm for graph realization. Math. Oper. Res. 1988, 13, 99-123. [CrossRef]

54. Ahuja, R.; Goldberg, A.; Orlin, J.; Tarjan, R. Finding minimum-cost flows by double scaling. Math. Program. 1992, 53, 243-266. [CrossRef]

55. Orlin, J. A faster strongly polynomial minimum cost flow algorithm. Oper. Res. 1993, 41, 338-350. [CrossRef] 
56. Kovácsab, P. Minimum-cost flow algorithms: An experimental evaluation. Opt. Methods Softw. 2015, 30, 94-127. [CrossRef]

57. Orlin, J.B. A polynomial time primal network simplex algorithm for minimum cost flows. Math. Program. 1997, 78, 109-129. [CrossRef]

58. MATLAB: R2017b; The MathWorks, Inc.: Natick, MA, USA, 2017.

59. Tounsi, M.; Mahlous, A.R. A Multi-Objective Model for Optimizing Network lifetime in Wireless Sensor Network. Int. J. Comput. Sci. Inf. Secur. 2016, 14, 562-569.

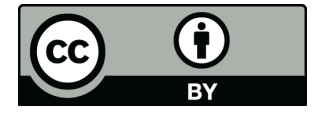

(c) 2017 by the author. Licensee MDPI, Basel, Switzerland. This article is an open access article distributed under the terms and conditions of the Creative Commons Attribution (CC BY) license (http://creativecommons.org/licenses/by/4.0/). 